\title{
CORREÇÃO DE ANEURISMA DE AORTA ABDOMINAL EM PACIENTE COM DOIS RINS PÉLVICOS
}

\section{ABDOMINAL AORTIC ANEURYSM REPAIR IN PATIENT WITH TWO PELVIC KIDNEYS}

\section{Luís Felipe da Silva, TCBC-RJ' ${ }^{1}$ André Luiz Fernandes²; Warley Dias Siqueira Mendes, ACBC-RJ ${ }^{3}$}

\section{INTRODUÇÃO}

A presença de um rim situado em posição pélvica é rara. Sua associação com aneurisma de aorta constitui situação mais rara ainda, em que a principal dificuldade técnica encontrada é a preservação da função renal durante o clampeamento aórtico ${ }^{1-5}$.

O rim pélvico pode ser congênito ou decorrente de transplante e a origem anormal da artéria renal pode acarretar maiores dificuldades técnicas ${ }^{1-4}$

O objetivo deste trabalho é relatar o caso de um paciente com dois rins pélvicos associados a aneurisma da aorta abdominal (AAA) tratado com sucesso, sem alteração da função renal.

\section{RELATO DO CASO}

Paciente de 65 anos, sexo feminino, branca, natural do Rio de Janeiro, tabagista de 25 maços/ano. Encaminhada ao Serviço de Cirurgia Vascular do Hospital Universitário Clementino Fraga Filho (HUCFF) com a suspeita diagnóstica de aneurisma da aorta abdominal. A paciente apresentava-se assintomática e ao exame físico observou-se, à palpação abdominal, massa pulsátil, expansiva e indolor, de cerca de $5 \mathrm{~cm}$ em mesogástrio. A tomografia computadorizada mostrou a presença de AAA de 5,6 cm em sua maior extensão, com rins situados em posição pélvica. Indicado estudo angiográfico da aorta abdominal que demonstrou artérias renais únicas bilateralmente, em posição ectópica, e dilatação aneurismática da aorta abdominal que se iniciava acima da emergência das artérias renais. A artéria renal direita tinha origem na aorta distal (Figura 1A) e a artéria renal esquerda na artéria ilíaca comum esquerda (Figura 1B). Ambos os rins estavam situados em região pélvica e deslocados para a direita.

Proposto o procedimento cirúrgico, este foi realizado sob anestesia geral, com incisão longitudinal, paramediana à esquerda, com acesso extraperitonial. Após a dissecção da aorta e reparo proximal e distal ao aneurisma, foi realizado sob heparinização sistêmica, na dose de $100 \mathrm{UI} / \mathrm{Kg}$, aneurismotomia e interposição aorto-aórtica com enxerto tubular do tipo poliéster com colágeno (Hemashield) de $18 \mathrm{~mm}$ de diâmetro. $\mathrm{O}$ tempo de clampeamento aórtico supra-renal foi de $45 \mathrm{~min}$. sem proteção renal específica e a anastomose realizada em chuleio com polipropileno 3-0 para as anastomoses proximal e distal. Após o desclampeamento aórtico observou-se diurese e os resultados dos exames laboratoriais no pós-operatório mostraram a preservação da função renal.

A paciente teve boa evolução clínica recebendo alta do Centro de Terapia Intensiva após 36 horas e alta hospitalar no $7^{\mathrm{o}}$ dia de pós-operatório.
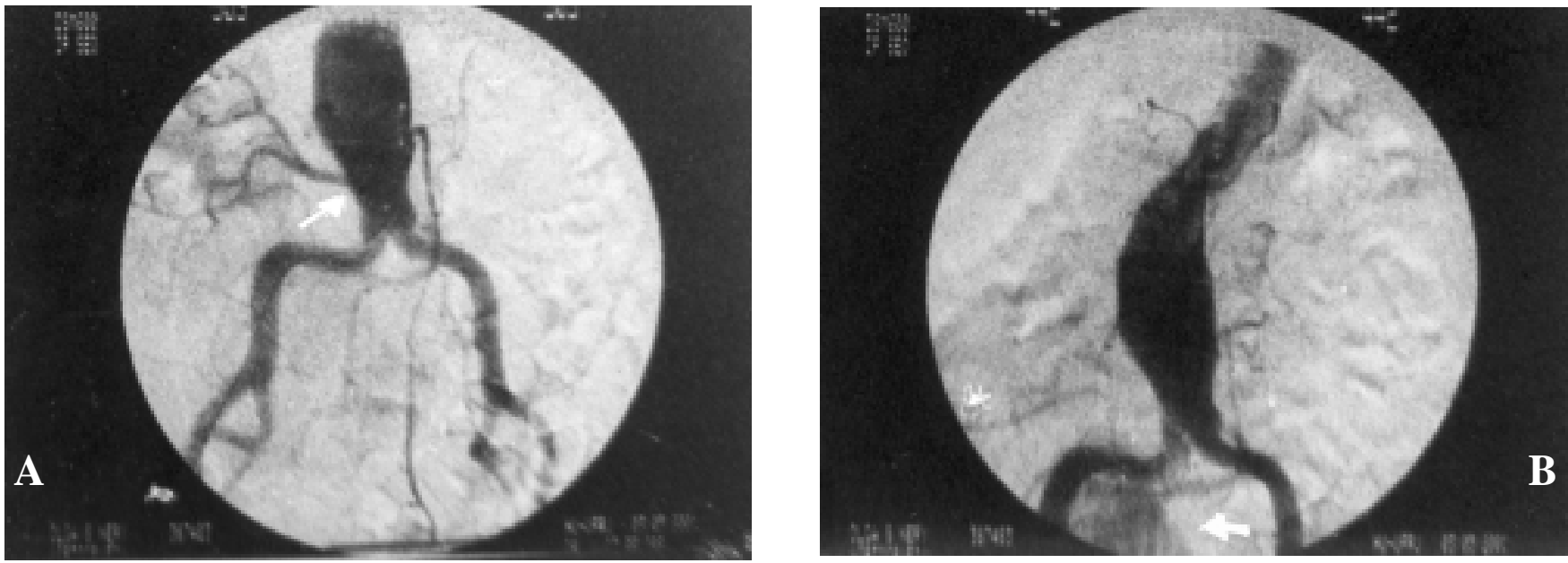

Figura 1 - (A) Angiografia da aorta abdominal distal evidenciando dilatação aneurismática supra-renal e artéria renal direita com origem no limite inferior do aneurisma (seta). (B) Angiografia da aorta abdominal evidenciando artéria renal esquerda derivando da artéria ilíaca comum esquerda, próxima à sua origem. Pode-se observar também, a presença do rim em região pélvica (seta).

1. Chefe do Departamento de Cirurgia da Faculdade de Medicina da UFRJ; Professor Adjunto do Departamento de Cirurgia da Faculdade de Medicina da UFRJ; Cirurgião do Serviço de Cirurgia Vascular do HUCFF.

2. Chefe do Serviço de Cirurgia Vascular do HUCFF; Professor Assistente do Departamento de Cirurgia da Faculdade de Medicina da UFRJ.

3. Mestrando em Cirurgia Geral, área de concentração Cirurgia Vascular, da Faculdade de Medicina da UFRJ.

Recebido em 19/12/2003

Aceito para publicação em 16/03/2004

Trabalho realizado no Departamento de Cirurgia da Faculdade de Medicina e Serviço de Cirurgia Vascular do Hospital Universitário Clementino Fraga Filho (HUCFF) da Universidade Federal do Rio de Janeiro (UFRJ). 


\section{DISCUSSÃo}

A incidência de um rim situado em posição pélvica é rara e sua associação com AAA é ainda mais ${ }^{1}$. Denomina-se rim pélvico aquele situado em região pélvica, seja congênito ou decorrente de transplante renal. Estima-se que a incidência de rim pélvico congênito seja de 1:2100 a 1:3000 nascimentos ${ }^{3}$. Embriologicamente o rim pélvico congênito resulta de uma falha da ascensão renal entre a $4^{\mathrm{a}}$ e $8^{\mathrm{a}}$ semanas de gestação, é geralmente menor e mantém lobulação fetal. Localiza-se mais comumente à esquerda, em situação ectópica mais baixa e apresenta ureter curto de entrada homolateral na bexiga com orifício intravesical de apresentação normal. A vascularização consiste de uma ou duas artérias renais que têm origem anormal na aorta distal, na bifurcação aórtica, na artéria ilíaca comum ou na artéria ilíaca externa ${ }^{3,4}$.

A maioria das reconstruções aórticas associadas com rim pélvico relatadas na literatura, foram realizadas em receptores de transplante renal que desenvolveram $\mathrm{AAA}^{3}$.

No caso relatado neste trabalho foram encontrados dois rins pélvicos congênitos, um com artéria renal originada na aorta distal e o outro na artéria ilíaca comum esquerda, e ambos os rins deslocados à direita.

$\mathrm{O}$ tratamento cirúrgico de AAA em pacientes com rim pélvico pode ser um procedimento difícil, não só pela particularidade da cirurgia de AAA mas também pelo alto risco de isquemia com dano irreversível ao rim. O tempo de isquemia que o rim humano pode tolerar ainda não é conhecido. Entretanto, acredita-se que um tempo de isquemia maior que 30 minutos em condições normotérmicas, pode promover dano no parênquima normal com necrose tubular aguda ${ }^{2}$.

A incidência de insuficiência renal aguda em cirurgias aórticas eletivas é de 1 a $8 \%$ mas nos aneurismas de aorta supra-renais, torácicos, justa-renais e rotos, pode alcançar $40 \%$. Os fatores predisponentes durante a cirurgia são a hipotensão arterial, politransfusões, embolização da artéria renal e mioglobinemia, sendo a causa mais importante a agressão isquêmica e seu tempo de duração.

A proteção renal durante a cirurgia de correção de AAA associado a rim pélvico é o interesse maior do cirurgião e do anestesista ${ }^{2}$. Entretanto, existem autores que afirmam que durante a cirurgia não são necessárias medidas específicas para proteção do rim, devido à perfusão retrógrada contínua, apesar de baixa pressão, proveniente das artérias lombares, mesentérica inferior e ilíacas ${ }^{2}$. A desvantagem é o fato de que não se pode prever com acerto em todos os pacientes o tempo de clampeamento aórtico, que pode exceder em muito o tempo suposto de segurança e promover lesão renal irreversível.

Várias técnicas têm sido usadas para proteção renal, porém, nenhuma foi considerada superior e mais segura para a preservação da função renal durante o clampeamento: "shunt" temporário ou "bypass" aorto-ilíaco, ou aorto-femoral, ou axilofemoral, hipotermia geral, perfusão renal com Ringer a $4^{\circ} \mathrm{C}$, resfriamento tópico, circulação extracorpórea ${ }^{1-4}$.

O manejo endovascular do AAA utilizando uma endoprótese é uma opção de tratamento menos agressiva, porém, que requer um planejamento pré-procedimento extremamente cuidadoso no momento de se calcular as medidas e fazer o desenho da prótese, ao traçar as manobras endoluminais a serem utilizadas e na forma de liberação da prótese.

No nosso caso, não foi utilizado nenhuma técnica específica para proteção renal, a não ser tentar realizar a parte principal da cirurgia no menor tempo possível para reduzir o tempo de isquemia renal.

\begin{abstract}
Abdominal aortic aneurysm reconstruction is usually performed in vascular surgical practice. However, the repair of an abdominal aortic aneurysm associated with a pelvic kidney is rare. Our goal is to present a case report of an abdominal aortic aneurysm associated with two congenital pelvic kidneys wich was treated successfully by aneurysmectomy and inclusion of an aortoaortic graft (Rev. Col. Bras. Cir. 2005; 32(1): 54-55).
\end{abstract}

Key words: Aortic aneurysm, abdominal; Kidney; Vascular surgical procedure.

\section{REFERÊNCIAS}

1. Belcastro S, Azzena GF, Gorini P, et al. - Abdominal aortic aneurysmectomy associated with a native iliac kidney. Case report. J Cardiovasc Surg, 1993,34(1):83-84.

2. Waikar HD, Neelakandhan KS, Ravimandalam K - Abdominal aortic aneurysmectomy in a patient with bilateral renal autotransplants. J Cardiothorac Vasc Anesth, 1995, 9(1):6670 .

3. Hollis HW, Rutherford RB, Crawford GJ, et al. - Abdominal aortic aneurysm repair in patients with pelvic kidney. Technical considerations and literature review. J Vasc Surg, 1989, 9(3):404409.

4. Glock Y, Blasevich R, Laghzaoui A, et al. - Abdominal aortic aneurysm and congenital pelvic kidney. A rare association. Tex Heart Inst J, 1997,24(2):131-133.
5. Ezzet F, Dorazio R, Herzberg R - Horseshoe and pelvic kidneys associated with abdominal aortic aneurysms. Am J Surg, 1977,134(2):196-198.

Endereço para correspondência:

Luís Felipe da Silva

Hospital Universitário Clementino Fraga Filho

Serviço de Cirurgia Vascular

Av. Brigadeiro Trompowsky s $/ \mathrm{n}^{\circ}$ - sala $10 \mathrm{E} 12$ - (10 $10^{\circ}$ andar $)$

Ilha do Fundão

CEP: 21941-590

- Tel.: (21)2562-2701 - Fax: (21)2562-2222

E-mail: felipe@ hucff.ufrj.br 